\title{
Reduced Serotonin Transporter Availability Decreases Prefrontal Control of the Amygdala
}

\author{
Inge Volman, ${ }^{1,2}$ Lennart Verhagen, ${ }^{2}$ Hanneke E. M. den Ouden, ${ }^{2,3}$ Guillén Fernández, ${ }^{2,4}$ Mark Rijpkema, ${ }^{2,5}$ \\ Barbara Franke, ${ }^{2,6}$ Ivan Toni, ${ }^{2}$ and Karin Roelofs ${ }^{1,2}$ \\ ${ }^{1}$ Behavioural Science Institute, Radboud University Nijmegen, 6525 HR Nijmegen, The Netherlands, ${ }^{2}$ Donders Institute for Brain, Cognition and Behaviour, \\ Radboud University Nijmegen, 6525 EN Nijmegen, The Netherlands, ${ }^{3}$ Center for Neural Science, New York University, New York, New York 10003, and \\ Departments of ${ }^{4}$ Cognitive Neuroscience, ${ }^{5}$ Nuclear Medicine, and ${ }^{6}$ Human Genetics, Radboud University Nijmegen Medical Centre, 6525 GA Nijmegen, The \\ Netherlands
}

After a threatening event, the risk of developing social psychopathologies is increased in short-allele (s) carriers of the serotonin transporter gene. The amygdala becomes overresponsive to emotional stimuli, an effect that could be driven by local hypersensitivity or by reduced prefrontal regulation. This study distinguishes between these two hypotheses by using dynamic causal modeling of fMRI data acquired in a preselected cohort of human s-carriers and homozygous long-allele carriers. Increased amygdala activity in s-carriers originates from reduced prefrontal inhibitory regulation when social emotional behavior needs to be controlled, suggesting a mechanism for increased vulnerability to psychopathologies.

\section{Introduction}

The neurotransmitter serotonin plays an important role in regulating social and emotional processes (Canli and Lesch, 2007). Serotonergic signaling is affected by a serotonin transporter polymorphism (5-HTTLPR), of which short-allele (s) carriers (homozygous and heterozygous) show reduced serotonin transporter availability and serotonin reuptake compared with non-carriers (homozygous long-allele carriers) (Lesch et al., 1996). This genetic variation in s-carriers leads to a heightened amygdala response to social threat (Hariri et al., 2002; Heinz et al., 2005; Caspi et al., 2010) and increased risk for the development of social psychopathologies (Caspi et al., 2010). Stressful or traumatic events intensify this vulnerability (Caspi et al., 2003; Karg et al., 2011). The combination of genetic and environmental factors suggests two possible accounts of increased vulnerability (Pezawas et al., 2005; Canli and Lesch, 2007; Caspi et al., 2010; Hyde et al., 2011): s-carriers could have a heightened sensitivity to emotional stimuli or decreased top-down control over emotional processing. Distinguishing between these possible mechanisms is important to advance our understanding of human emotional processing and to target therapies toward either reducing emotional sensitivity or increasing emotional control (Hyde et al., 2011).

\footnotetext{
Received Nov. 30, 2012; revised April 12, 2013; accepted April 13, 2013

Author contributions: I.V., G.F., I.T., and K.R. designed research; I.V. and L.V. performed research; G.F., M.R., and B.F. contributed unpublished reagents/analytic tools; I.V., L.V., H.E.M.d.O., I.T., and K.R. analyzed data; I.V., L.V., H.E.M.d.O., I.T., and K.R. wrote the paper.

This work was supported by the Netherlands Organization for Scientific Research (VIDI Grant 452-07-008 to K.R. and VICI Grant 453-08-002 to I.T.).

The authors declare no competing financial interests.

Correspondence should be addressed to Inge Volman, Montessorilaan 3, Room A.07.18, 6525 HR Nijmegen, The Netherlands. E-mail: inge.volman@donders.ru.nl.

DOI:10.1523/JNEUROSCI.5518-12.2013

Copyright $\odot 2013$ the authors $\quad 0270-6474 / 13 / 338974-06 \$ 15.00 / 0$
}

This study used dynamic causal modeling (DCM) (Friston et al., 2003) for fMRI, an analytical framework enabling tests of directionality in brain connectivity, to dissociate between those two mechanisms. Emotional processing was assessed with the social approach-avoidance (AA) task (Roelofs et al., 2009; Volman et al., 2011b), a well established protocol measuring behavioral and neural responses during automatic and controlled actions to emotional faces (Fig. 1A). Automatic response tendencies resulting in the approach of positive stimuli and the avoidance of negative stimuli are thought to be triggered by the amygdala (Quirk and Gehlert, 2003). When these emotional action tendencies need to be controlled during the AA task, the anterior prefrontal cortex ( $\mathrm{aPFC}$ ) has been shown to coordinate the contributions of several brain regions, including the amygdala (Volman et al., 2011a). Experimental alterations of the aPFC reduce emotional control and increase reactivity to emotional faces in the amygdala and in the fusiform face area (FFA) (Volman et al., 2011a). Here we assessed how physiological alterations of serotonin transporter availability influence the dynamics of this network during the production of automatic and controlled actions to emotional faces: via altered sensitivity or prefrontal control.

\section{Materials and Methods}

\section{Participants}

A total of $24 \mathrm{~s}$-carriers (9 homozygous, 15 heterozygous) and 24 noncarriers were preselected with a double-blind design. All participants were right-handed male students with normal or corrected-to-normal vision and no history of neurological or psychiatric illness matched (between groups) for age (mean $\pm \mathrm{SD}, 23 \pm 2.6$ and $22 \pm 2.4$ years), anxiety trait inventory (Spielberger, 1983; $32 \pm 7.2$ and $32 \pm 5.4$ ), depression (Beck et al., 1979; $2.8 \pm 3.3$ and $3.9 \pm 3.2$ ), and traumatic experiences (Nijenhuis et al., 2002; $1.75 \pm 1.6$ and $1.79 \pm 1.6)\left(\right.$ all $p>0.2, F_{(1,46)}<$ 1.6). The first 20 participants were randomly selected and used in a related study (Volman et al., 2011b). The remaining participants were 


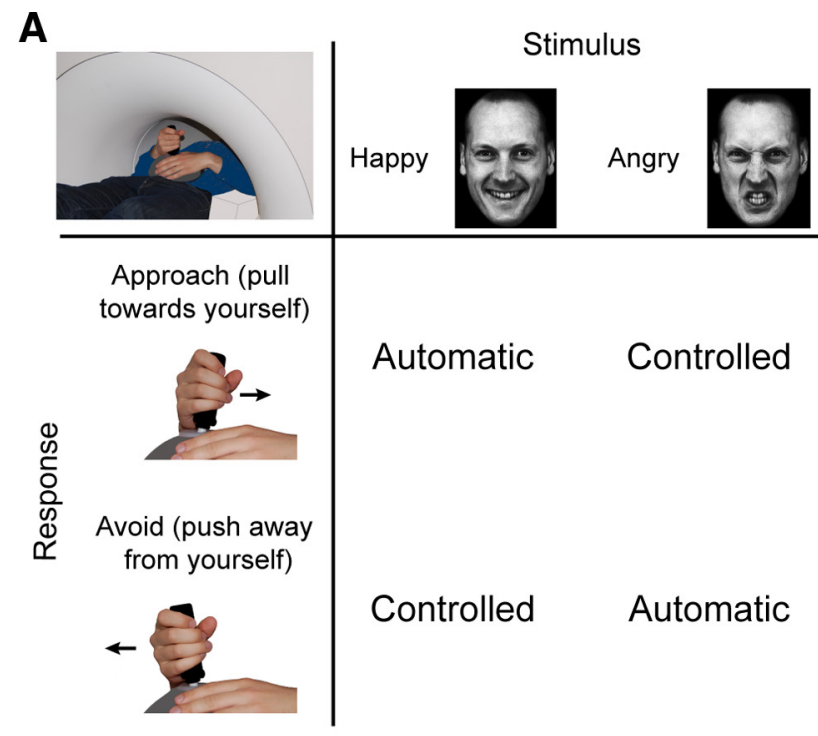
B Controlled > Automatic
口: Non-carriers
Amygdala
$\square$ : S-carriers
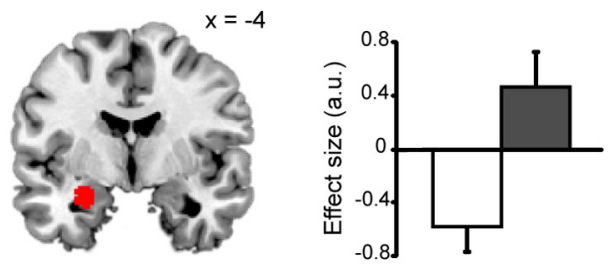

Figure 1. A, Design of the AA task. When viewing happy or angry faces, automatic stimulus-response tendencies trigger corresponding approach or avoidance actions. These tendencies could be followed during the automatic condition. In contrast, when task instructions required participants to avoid happy or approach angry faces, automatic tendencies needed to be controlled and overridden with the instructed response (controlled condition; Volman et al., 2011a). B, Regional effects of emotional control (controlled vs automatic trials) within amygdala (between-group differences) and aPFC (across groups). Left, SPM $\{t\}$ overlapped on a representative brain (thresholded at $p<0.05$ uncorrected for visualization). Right, Biased effect size of emotion control contrast \pm SEM of the clusters presented on the left.

preselected based on their 5-HTTLPR genotype. All participants provided written informed consent according to the guidelines of the local ethics committee.

\section{Procedure and experimental tasks}

The experimental setup, including tasks, salivary measurements, and imaging details, was as described previously (Volman et al., 2011b). Briefly, participants completed questionnaires and provided saliva, followed by the task-fMRI measurements, a resting-state scan (not reported here), and an anatomical scan. The AA task and an additional gender evaluation (GE) task were administered in two consecutive sessions (30 min each), with counterbalanced order across participants. Each task consisted of 24 blocks of 12 trials during which participants responded to visually presented faces by pulling a joystick toward themselves (approach) or by pushing it away from themselves (avoid). During the AA task, participants categorized faces as happy, angry, or neutral (filler items) based on their affective expressions. The combination of these emotion-response mappings introduced a distinction between more "automatic" conditions (approach-happy, avoid-angry) and "controlled" conditions (approach-angry, avoid-happy). We will refer to this factor as "emotional control": the additional processing required to overcome the prepotent response and to apply the counterintuitive rule. During the GE (control) task, participants categorized the gender (affectively irrelevant) of the same faces, thereby requiring no rule-driven action selection based on the emotion of the face (Roelofs et al., 2009). Each face was presented for $100 \mathrm{~ms}$, preceded by a $300 \mathrm{~ms}$ blank screen and followed by the participant's response, a blank screen, and a pseudorandom intertrial-interval (1-3 s). A baseline period of 21-24 s preceded each block.

\section{Materials and apparatus}

Functional MR images were acquired on a 1.5 T MRI scanner (Avanto; Siemens) with an 8-channel head coil using a multi-echo GRAPPA sequence (Poser et al., 2006) (TR 2.14 s, TEs 9.4/21/33/44/56 ms, 34 transversal slices, ascending acquisition, distance factor: $17 \%$, effective voxel size $3.3 \times 3.3 \times 3.5 \mathrm{~mm}$, FOV $212 \mathrm{~mm}$ ). Anatomical MR images were acquired using a magnetization prepared rapid gradient echo sequence (TR $2250 \mathrm{~ms}$, TE $2.95 \mathrm{~ms}, 176$ sagittal slices, voxel size $1 \times 1 \times 1 \mathrm{~mm}$, FOV $256 \mathrm{~mm}$ ).

\section{DNA analysis}

DNA was isolated from saliva using the Oragene system (DNA Genotek). Genotyping of the 5-HTTLPR polymorphism in the SLC6A4 (5-HTT, $S E R T$ ) gene was performed by simple sequence length analysis. PCR was on $50 \mathrm{ng}$ of genomic DNA using $0.5 \mu \mathrm{m}$ fluorescently labeled forward primer (FAM-5'-GGCGTTGCCGCTCTGAATGC- $3^{\prime}$ ) and reverse primer (5'-GAGGGACTGAGCTGGACAACCAC-3'), 0.25 mm dNTPs, $1 \times$ PCR optimization buffer A [30 mm Tris- $\mathrm{HCl}, \mathrm{pH} 8.5,7.5 \mathrm{~mm}$ $\left(\mathrm{NH}_{4}\right)_{2} \mathrm{SO}_{4}, 0.75 \mathrm{~mm} \mathrm{MgCl}_{2}$ ], 10\% DMSO, and $0.4 \mathrm{U}$ of AmpliTaq Gold DNA-polymerase (Applied Biosystems). The cycling conditions for the PCR were $12 \mathrm{~min}$ at $95^{\circ} \mathrm{C}, 35$ cycles of $1 \mathrm{~min}$ at $94^{\circ} \mathrm{C}, 1 \mathrm{~min}$ at the optimized annealing temperature $\left(57.5^{\circ} \mathrm{C}\right), 2 \mathrm{~min}$ at $72^{\circ} \mathrm{C}$, followed by 10 min at $72^{\circ} \mathrm{C}$. For quality control, $5 \%$ blanks and duplicates between plates were also taken. Determination of allele length was by automated capillary sequencing (ABI3730; Applied Biosystems).

\section{Behavioral analysis}

Trials in which the joystick was moved in the wrong direction or those showing an extreme reaction time (RT; $<100$ or $>1500 \mathrm{~ms}$ ), joystick peak velocity or path length $(>3 \mathrm{SD}$ from subject-specific data distribution) were excluded (Volman et al., 2011b). If the error rate of a block was above chance level, the whole block was excluded. Median RTs were calculated and entered in a 4-way repeated-measures ANOVA (ANOVA$\mathrm{rm})$, with factors genotype (s-carriers, non-carriers), task (AA, GE), movement (approach, avoid), and valence (happy, angry). The $a$-level was set at $p<0.05$.

\section{fMRI data: regional effects analyses}

The imaging data were preprocessed as described previously (Volman et al., 2011b). Participant-specific fMRI time series were analyzed using a general linear model including the following effects for each task (AA, GE) separately: approach-happy, approach-neutral, approach-angry, avoid-happy, avoid-neutral, and avoid-angry. Trials excluded from behavioral analyses and instruction periods were modeled with separate regressors. The group-level random effects multiple regression analysis considered 16 conditions, given by the combination of four factors: genotype (s-carriers, non-carriers), task (AA, GE), valence (happy, angry), and condition (automatic, controlled), with testosterone and cortisol levels as condition-specific covariates (Volman et al., 2011b). The regions 
of interest (ROIs) followed our hypotheses, namely amygdala from the aal atlas (Tzourio-Mazoyer et al., 2002) using the WFU PickAtlas tool (Maldjian et al., 2003) and aPFC from Volman et al. (2011a, 2011b) (two spheres; radius: $8 \mathrm{~mm}$; centers: $-30,58,2$ and 32, 54, 8). Correction for multiple comparisons was implemented with small volume familywise error (FWE).

\section{fMRI data: DCM}

Time series extraction. $\mathrm{fMRI}$ time series representative of the left FFA, left amygdala, and left aPFC (Volman et al., 2011a) were selected on ROIs defined with anatomical and functional constraints (Stephan et al., 2010). The left aPFC anatomical search space was described above (regional effects analysis). The FFA space was a sphere (radius: $10 \mathrm{~mm}$; center: $-43,-54,-17$ ) defined by a previous mapping study (Liu et al., 2010). The amygdala space was based on anatomical information obtained from individual MRI scans (FSL-FIRST v1.2; http://www.fmrib. ox.ac.uk/fsl/first; Patenaude et al., 2011). For each ROI in each participant, 20 voxels were selected that showed the strongest effect on an $F$ test on main effect of happy and angry faces during the AA task from the first level model with an explicit baseline. The first eigenvariate across these voxels was extracted after removing variance explained by effects of no interest.

DCM model specification. Analysis of regional fMRI effects indicated increased aPFC responses across both genotypes and increased amygdala activity in s-carriers compared with non-carriers during emotional control (see Results). DCM was used to distinguish between the two hypothesized mechanisms accounting for the different amygdala response to emotional control in the two genotypes. We defined the basic model structure common across all models (Fig. $2 A$ ): presentation of a face as driving input in the FFA node (with RT as duration), forward projection from FFA to amygdala (Pessoa et al., 2002; Gschwind et al., 2012), and a bidirectional connection between amygdala and aPFC, after evidence of functional and anatomical connections (Bracht et al., 2009; Volman et al., 2011b).

Given this basic model, we tried to identify where emotional control modulated or enabled extrinsic or self-connections within this distributed system. Our model space addressed two key questions: (1) how emotional control modulated the aPFC response and (2) whether additional control-related changes in connection strengths were necessary to explain amygdala responses. This resulted in a factorial model space in which the first factor had four levels: emotional control changed the amygdala $\rightarrow$ aPFC connection, the aPFC self-connection, an additional FFA $\rightarrow$ aPFC connection, or it excited the prefrontal cortex directly. The second factor investigated whether the source of the amygdala modulation originated from the aPFC or from another unspecified region (Stephan et al., 2008). This factor had three levels with six variations in total: (1) a basic model (aPFC influences amygdala via aPFC $\rightarrow$ amygdala connection), (2) aPFC activity modulates FFA $\rightarrow$ amygdala connection and amygdala self-connection, or (3) emotional control modulates FFA $\rightarrow$ amygdala connection, amygdala self-connection, and acts as driving input on the amygdala. This model space allowed us to address the generation of differential responses in the aPFC by comparing families of models distinguished by the first factor (averaging over the second). These correspond to prefrontal families A-D in Figure 2C. Finally, we were able to address our key question about the prefrontal control of amygdala responses by comparing models with and without further emotion control effects. These are amygdala families A-C in Fig. 2C.

Model inference. For each factor, we tested which family of models best explained the data across all participants using the negative free energy approximation to log model evidence (Friston and Stephan, 2007). A random-effects Bayesian model selection procedure for model families (Penny et al., 2010) was used to derive the exceedance probability $\left(\mathrm{XP}_{\mathrm{k}}\right.$; i.e., the probability that a particular model family $\mathrm{k}$ is more likely than any other model family considered, given the data). We also checked for differences in the optimal model or model ranking between genotype groups.

Parameter inference. We then compared the parameters of the winning families to test for genotype differences in emotional control of the amygdala. When it was not possible based on the model evidence to distinguish between two families $\left(\mathrm{XP}_{\mathrm{k}}>0.95\right)$, Bayesian model averaging across all families with $\mathrm{XP}_{\mathrm{k}}>0.05$ over genotypes was applied (Penny et al., 2010). Bayesian model averaging calculates an average parameter estimate for each connection and participant across a set of models, weighted by the posterior probability of each model. This procedure enables inference about model parameters while accounting for differences in model evidence. Next, a univariate GLM was created for each of the parameter estimates modeling emotional control to the amygdala. The reciprocal SD of that estimate was considered as a weight for leastsquares regression to adjust for heteroscedasticity (Carroll and Ruppert, 1988). One outlier was present and removed (post hoc analyses with outlier produced similar results: genotype effect on aPFC $\rightarrow$ amygdala: $\left.F_{(1,46)}=12.5, p=0.001\right)$. The $\alpha$-level was Bonferroni corrected $(0.05 /$ $3=0.017)$.

\section{Results}

\section{Behavioral data}

Participants performed the tasks accurately (omissions: $0.9 \%$; error rate: $6.8 \%$; trials excluded due to block errors: $0.4 \%$; anomalous kinematic responses: $5.8 \%$ ) and consistently (Table 1). The 4-way ANOVA-rm showed a main effect of task $\left(F_{(1,46)}=22.8\right.$, $p<0.001)$, movement $\left(F_{(1,46)}=4.2, p=0.047\right)$, valence $\left(F_{(2,45)}=\right.$ 139.2, $p<0.001)$, task $\times$ valence interaction $\left(F_{(2,45)}=24.5, p<\right.$ 0.001 ), and an emotional control effect over both tasks (movement $\times$ valence; $\left.F_{(1,46)}=6.1, p=0.017\right)$. When considering each task separately, both tasks showed a valence effect (AA task: $F_{(1,46)}=$ 96.7, $p<0.001$, GE task: $\left.F_{(1,46)}=26.2, p<0.001\right)$. Only the AA task showed an emotional control effect $\left(F_{(1,46)}=4.9, p=0.032 ; \mathrm{GE}\right.$ task: $p=0.228$ ), as also found previously (Roelofs et al., 2009), indicating that controlled conditions evoked significantly longer RTs than automatic conditions. There was no evidence for genotype differences in $\mathrm{RT}$ or error rate (all $p>0.05$ ).

\section{fMRI data: regional effects analyses}

First, the amygdala showed a genotype (s-carriers $>$ non-carriers) $\times$ condition (controlled $>$ automatic) trend effect for the AA task (left side; $z=3.09, p_{\mathrm{FWE}}=0.061$, coordinates of local maxima: $-28,-4,-20$, Fig. $1 B)$. Post hoc testing indicated that this effect was mainly driven by angry faces $\left(z=3.19, p_{\mathrm{FWE}}=0.046\right.$, local maxima: $-28,-4,-20)$ and less by happy faces $(z=2.00$, $p_{\text {uncorrected }}<0.05$, local maxima: $\left.-26,-6,-18\right)$. When masking the interaction with the three-way interaction (genotype [s-carriers $>$ non-carriers] $\times$ task $[\mathrm{AA}>\mathrm{GE}] \times$ condition [controlled $>$ automatic]), the effects did not change, indicating the results are specific for the AA task. Second, the left and right aPFC (BA 10) showed a stronger response for the controlled than for the automatic trials during the AA task $\left(z=3.47, p_{\mathrm{FWE}}=0.021\right.$, local maxima: $-30,60,6$, and $z=3.50, p_{\mathrm{FWE}}=0.019$, local maxima: 32, 52, 6, respectively; Fig. $1 B$ ). When comparing the effects of the AA task with that of the GE task (by masking the contrast with the two-way interaction [task $(\mathrm{AA}>\mathrm{GE}) \times$ condition (controlled $>$ automatic)] ), the effects remained the same. This indicates that the results are specific to the (explicit) AA task, replicating previous reports on these tasks (Roelofs et al., 2009; Volman et al., 2011b).

\section{fMRI data: DCM}

Formal model comparison indicated that, in the best models across genotypes, emotional control modulated the aPFC both via self-connection and amygdala feedforward input, whereas the amygdala was modulated via the aPFC (Fig. $2 D, E$ ).

Crucially, the strength of the direct aPFC $\rightarrow$ amygdala connection showed a significant genotype difference $\left(F_{(1,45)}=6.8, p=\right.$ 0.012 , Fig. $2 E)$. This aPFC $\rightarrow$ amygdala connection had a negative 
A Basic model

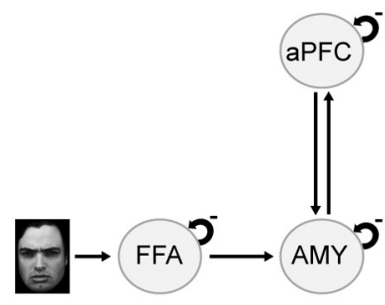

B Potential mechanisms of emotional control (EC) factor 1: in aPFC factor 2: in amygdala
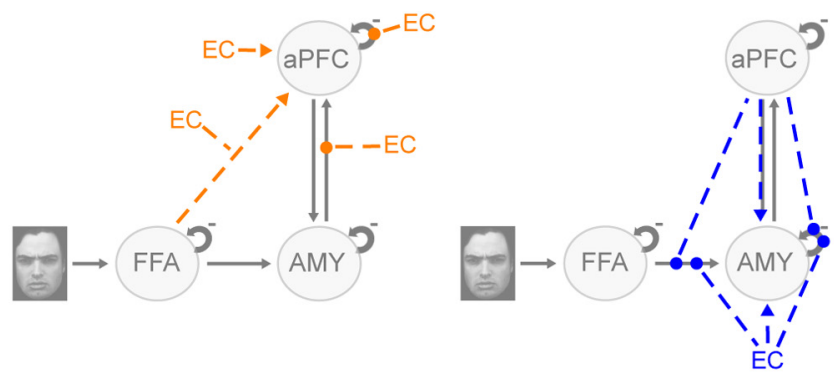

C Schematic overview of all models

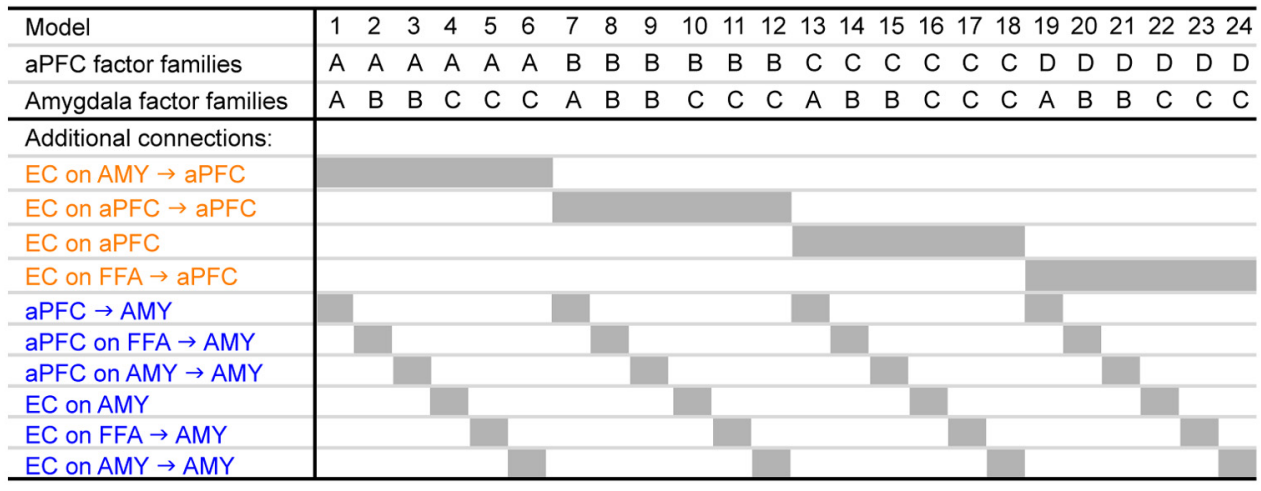

D Results of model selection

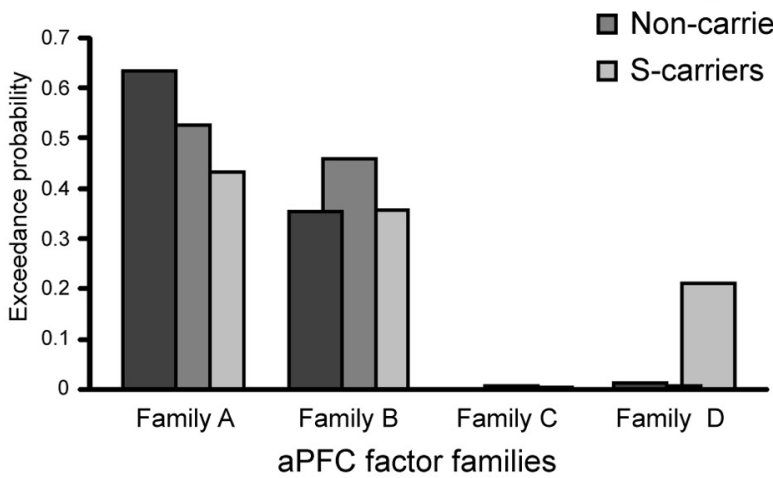

Over genotypes

Non-carriers

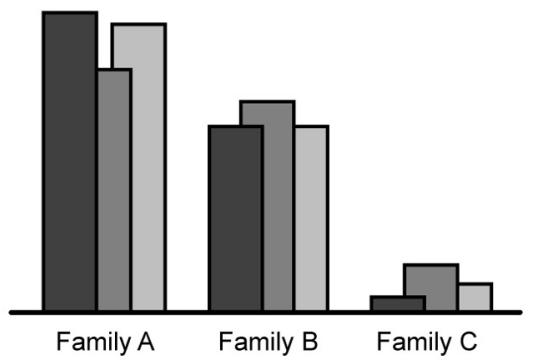

Amygdala factor families
E Winning models (summary)

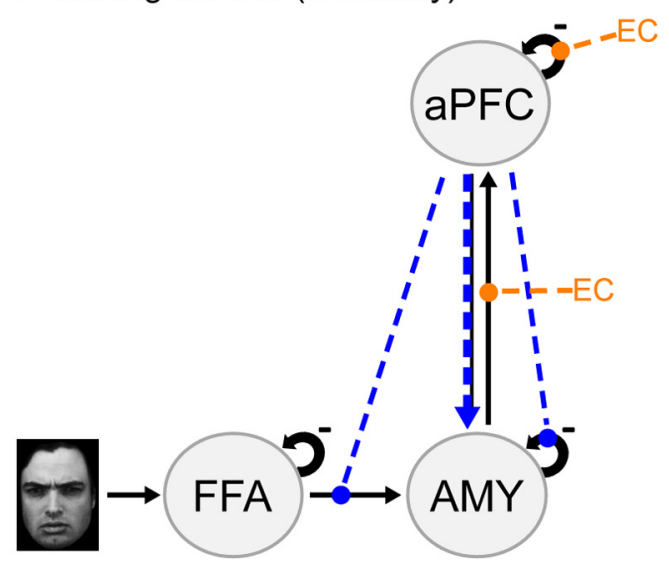

F Effect of genotype

$\square$ : Non-carriers

ㅁ: S-carriers

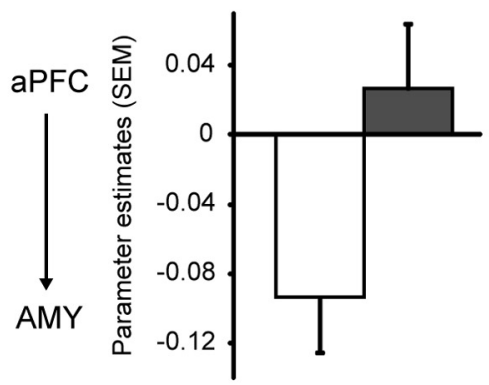

Figure 2. Modulation of interregional connectivity. $A$, Basic structure of the model: face presentation drives FFA activity, which projects to the amygdala (AMY) that is bidirectionally connected with aPFC. B, Emotional control could modulate aPFC (orange) and amygdala activity (blue) either directly, on connections, or via other nodes. $C$, Overview representing all 24 models and their corresponding families within each factor. Each model contained the basic model with one orange and one blue connection. $\boldsymbol{D}, \mathrm{XP}_{\mathrm{k}}$ for each factor of the model selection (Figure legend continues.) 
Table 1. Mean RTs (SEM) in milliseconds

\begin{tabular}{llllll}
\hline & S-carriers & & & \multicolumn{1}{l}{ Non-carriers } & \\
& Approach & Avoid & & Approach & Avoid \\
\hline AA-task & & & & \\
$\quad$ Happy & $524(18)$ & $554(20)$ & & $517(19)$ & $546(21)$ \\
$\quad$ Neutral & $592(19)$ & $598(18)$ & & $564(20)$ & $583(21)$ \\
$\quad$ Angry & $587(19)$ & $583(18)$ & & $575(20)$ & $587(20)$ \\
$\begin{array}{c}\text { GE-task } \\
\text { Happy }\end{array}$ & $509(12)$ & $530(17)$ & & $511(15)$ & $525(15)$ \\
Neutral & $515(13)$ & $528(16)$ & & $509(16)$ & $524(16)$ \\
Angry & $535(14)$ & $543(17)$ & & $526(16)$ & $539(16)$ \\
\hline
\end{tabular}

Table 2. DCM parameters showing the estimated mean (SEM) in Hertz

\begin{tabular}{lccc}
\hline Parameter & Over genotypes & \multicolumn{1}{c}{ Non-carriers } & \multicolumn{1}{c}{$s$-carriers } \\
\hline Faces to FFA & $0.08(0.07)$ & $0.10(0.11)$ & $0.07(0.10)$ \\
FFA $\rightarrow$ AMY & $0.07(0.03)$ & $0.08(0.04)$ & $0.07(0.05)$ \\
AMY $\rightarrow$ aPFC & $-0.01(0.05)$ & $-0.05(0.08)$ & $0.02(0.07)$ \\
aPFC $\rightarrow$ AMY & $-0.04(0.03)$ & $-0.09(0.03)$ & $0.03(0.04)$ \\
aPFC on FFA $\rightarrow$ AMY & $-0.003(0.002)$ & $-0.003(0.003)$ & $-0.003(0.002)$ \\
aPFC on AMY $\rightarrow$ AMY & $-0.001(0.001)$ & $-0.002(0.002)$ & $-0.0004(0.002)$ \\
C on AMY $\rightarrow$ aPFC & $0.02(0.01)$ & $0.02(0.02)$ & $0.02(0.01)$ \\
Con aPFC $\rightarrow$ aPFC & $0.01(0.01)$ & $-0.004(0.004)$ & $0.02(0.01)$ \\
\hline
\end{tabular}

weight for non-carriers $\left(F_{(1,23)}=10.6, p=0.003\right)$, but was not significantly different from zero for $s$-carriers $\left(F_{(1,22)}=0.5, p=\right.$ 0.473; Table 2). Indirect top-down aPFC modulations of the amygdala (amygdala factor family B) showed no group difference (both $F<0.9, p>0.3$ ).

In summary, the best explanation for control-related effects in the aPFC was an increase in amygdala $\rightarrow$ aPFC connectivity and aPFC self-connectivity. The subsequent prefrontal, controlrelated responses are then communicated back to the amygdala (with no need for emotional control modulations from other sources). However, the inhibitory aPFC $\rightarrow$ amygdala control effects are reduced in s-carriers, leading to disinhibited emotional amygdala responses.

\section{Discussion}

Genetic differences associated with altered serotonin function influence functional and anatomical connectivity between the amygdala and ventral PFC (Heinz et al., 2005; Pezawas et al., 2005; Holmes, 2008; Pacheco et al., 2009). This study qualifies that alteration, showing that serotonin influences the ability of the aPFC to control amygdala reactivity to emotional faces. In $s$-carriers, automatic amygdala responses are not adequately inhibited by the aPFC when emotional control is required to facilitate an alternative course of action. The specificity of these connectivity effects was further qualified by analyses of regional activity and behavioral performance.

In this study, participants followed either their automatic response tendency to approach happy and avoid angry faces or they had to override that tendency with the opposite counterintuitive

$\leftarrow$

(Figure legend continued.) for model families. For the aPFC factor, families 1 and 2 together show $X P_{k}>0.95$. For the amygdala factor, $X P_{k}>0.95$ is reached for families 1 and 2 together, all containing a modulation via the $\mathrm{APFC}$. These $\mathrm{XP}_{\mathrm{k}}$ values are similar for non-carriers (medium gray), s-carriers (light gray), and across groups (dark gray). $E$, Best models (combined $X P_{k}>$ 0.95) for the fMRI data across genotypes in which emotional control modulated the aPFC and aPFC modulated the amygdala. These models were averaged within subjects for parameter inference. $\boldsymbol{F}$, Parameter estimates ( \pm SEM) of the direct aPFC $\rightarrow$ amygdala connection. Noncarriers, but not s-carriers, showed significant inhibition of amygdala by the aPFC. stimulus-response mapping (happy-avoid, angry-approach). The amygdala is thought to trigger automatic emotional response tendencies, whereas during emotional control, the aPFC is suggested to coordinate the involvement of several brain regions, including the amygdala (Quirk and Gehlert, 2003; Volman et al., 2011a). Exerting emotional control evoked stronger amygdala activity in s-carriers, whereas aPFC activity and behavioral performance was comparable across groups. These findings indicate that reduced aPFC inhibition in s-carriers was not a by-product of between-group differences in aPFC activity or behavior. They also provide evidence that reduced inhibitory coupling from aPFC to amygdala in s-carriers can explain enhanced amygdala activity when emotional control is required. Finally, these effects were specific for emotional control: they were significantly stronger in the AA task compared with an additional task with the same stimuli and emotionally irrelevant instructions (Roelofs et al., 2009).

A strong emotional event such as stress or trauma results in a strong amygdala response, increasing the bias for automatic emotional behavior (Quirk and Gehlert, 2003). In s-carriers, a lack of prefrontal control reduces the possibility of inhibiting these biases. The enhanced expression of automatic tendencies explained by this connectivity-based mechanism could account for the increased stress sensitivity and vulnerability to social psychopathologies observed in s-carriers, but also their heightened sensitivity for all motivationally relevant inputs (Lesch et al., 1996; Canli and Lesch, 2007; Caspi et al., 2010; Homberg and Lesch, 2011).

The present results suggest that anterior prefrontal inhibition of the amygdala is an important mechanism during control of social emotional actions, and a reduction thereof is a risk factor for the development of social psychopathologies. Several studies focused on assessing the neural mechanisms of a diverse range of psychopathologies support this theory, showing that prefrontal-amygdala coupling was affected during emotional processing (Kim et al., 2011; Rudie et al., 2012; Strakowski et al., 2012). A recent DCM study even demonstrated reduced effective connectivity from the PFC to the amygdala in patients with depression (Almeida et al., 2011) often associated with serotonin dysfunction (Holmes, 2008). Furthermore, humans and animals studies that focus on lesions of the aPFC and the adjacent orbital frontal cortex reported reduced control of social and emotional behavior (Damasio et al., 1994; Berlin et al., 2004; Agustín-Pavón et al., 2012). Reduced functionality of the aPFC induced by transcranial magnetic stimulation also decreased the ability to apply emotional control while at the same time enhancing activity of the amygdala (Volman et al., 2011a).

To conclude, this effective connectivity study shows that a lack of prefrontal inhibitory regulation underlies the increased amygdala response in individuals with reduced serotonin reuptake (s-carriers) during control of social emotional behavior. This result suggests that increasing emotional control would be an effective therapeutic intervention for preventing the emergence of social psychopathologies in s-carriers. For example, explicitly instructed emotion regulation strategies can reduce 5-HTTLPR-related amygdala differences (Schardt et al., 2010). Moreover, improving the capacity to exert emotional control might be a (preventive) strategy for s-carriers in case they need to be prepared for challenging situations, for example, in stressful professions. 


\section{References}

Agustín-Pavón C, Braesicke K, Shiba Y, Santangelo AM, Mikheenko Y, Cockroft G, Asma F, Clarke H, Man MS, Roberts AC (2012) Lesions of ventrolateral prefrontal or anterior orbitofrontal cortex in primates heighten negative emotion. Biol Psychiatry 72:266-272. CrossRef Medline

Almeida JR, Kronhaus DM, Sibille EL, Langenecker SA, Versace A, Labarbara EJ, Phillips ML (2011) Abnormal left-sided orbitomedial prefrontal cortical-amygdala connectivity during happy and fear face processing: a potential neural mechanism of female MDD. Front Psychiatry 2:69. CrossRef Medline

Beck AT, Rush AJ, Hollon SD, Emery G (1979) Cognitive therapy of depression. New York: Wiley.

Berlin HA, Rolls ET, Kischka U (2004) Impulsivity, time perception, emotion and reinforcement sensitivity in patients with orbitofrontal cortex lesions. Brain 127:1108-1126. CrossRef Medline

Bracht T, Tüscher O, Schnell S, Kreher B, Rüsch N, Glauche V, Lieb K, Ebert D, Il'yasov KA, Hennig J, Weiller C, van Elst LT, Saur D (2009) Extraction of prefronto-amygdalar pathways by combining probability maps. Psychiatry Res 174:217-222. CrossRef Medline

Canli T, Lesch KP (2007) Long story short: the serotonin transporter in emotion regulation and social cognition. Nat Neurosci 10:1103-1109. CrossRef Medline

Carroll RJ, Ruppert D (1988) Transformation and Weighting in Regression: Chapman and Hall.

Caspi A, Sugden K, Moffitt TE, Taylor A, Craig IW, Harrington H, McClay J, Mill J, Martin J, Braithwaite A, Poulton R (2003) Influence of life stress on depression: moderation by a polymorphism in the 5-HTT gene. Science 301:386-389. CrossRef Medline

Caspi A, Hariri AR, Holmes A, Uher R, Moffitt TE (2010) Genetic sensitivity to the environment: the case of the serotonin transporter gene and its implications for studying complex diseases and traits. Am J Psychiatry 167:509-527. CrossRef Medline

Damasio H, Grabowski T, Frank R, Galaburda AM, Damasio AR (1994) The return of Phineas Gage: clues about the brain from the skull of a famous patient. Science 264:1102-1105. CrossRef Medline

Friston KJ, Stephan KE (2007) Free-energy and the brain. Synthese 159: 417-458. CrossRef Medline

Friston KJ, Harrison L, Penny W (2003) Dynamic causal modelling. Neuroimage 19:1273-1302. CrossRef Medline

Gschwind M, Pourtois G, Schwartz S, Van De Ville D, Vuilleumier P (2012) White-matter connectivity between face-responsive regions in the human brain. Cereb Cortex 22:1564-1576. CrossRef Medline

Hariri AR, Mattay VS, Tessitore A, Kolachana B, Fera F, Goldman D, Egan MF, Weinberger DR (2002) Serotonin transporter genetic variation and the response of the human amygdala. Science 297:400-403. CrossRef Medline

Heinz A, Braus DF, Smolka MN, Wrase J, Puls I, Hermann D, Klein S, Grüsser SM, Flor H, Schumann G, Mann K, Buchel C (2005) Amygdalaprefrontal coupling depends on a genetic variation of the serotonin transporter. Nat Neurosci 8:20-21. CrossRef Medline

Holmes A (2008) Genetic variation in cortico-amygdala serotonin function and risk for stress-related disease. Neurosci Biobehav Rev 32:1293-1314. CrossRef Medline

Homberg JR, Lesch KP (2011) Looking on the bright side of serotonin transporter gene variation. Biol Psychiatry 69:513-519. CrossRef Medline

Hyde LW, Bogdan R, Hariri AR (2011) Understanding risk for psychopathology through imaging gene-environment interactions. Trends Cogn Sci 15:417-427. CrossRef Medline

Karg K, Burmeister M, Shedden K, Sen S (2011) The serotonin transporter promoter variant (5-HTTLPR), stress, and depression metaanalysis revisited: evidence of genetic moderation. Arch Gen Psychiatry 68:444-454. CrossRef Medline

Kim MJ, Loucks RA, Palmer AL, Brown AC, Solomon KM, Marchante AN, Whalen PJ (2011) The structural and functional connectivity of the amygdala: from normal emotion to pathological anxiety. Behav Brain Res 223:403-410. CrossRef Medline

Lesch KP, Bengel D, Heils A, Sabol SZ, Greenberg BD, Petri S, Benjamin J, Müller CR, Hamer DH, Murphy DL (1996) Association of anxiety- related traits with a polymorphism in the serotonin transporter gene regulatory region. Science 274:1527-1531. CrossRef Medline

Liu J, Harris A, Kanwisher N (2010) Perception of face parts and face configurations: an FMRI study. J Cogn Neurosci 22:203-211. CrossRef Medline

Maldjian JA, Laurienti PJ, Kraft RA, Burdette JH (2003) An automated method for neuroanatomic and cytoarchitectonic atlas-based interrogation of fMRI data sets. Neuroimage 19:1233-1239. CrossRef Medline

Nijenhuis ERS, Van der Hart O, Kruger K (2002) The psychometric characteristics of the Traumatic Experiences Checklist (TEC): first findings among psychiatric outpatients. Clin Psychol Psychot 9:200-210. CrossRef

Pacheco J, Beevers CG, Benavides C, McGeary J, Stice E, Schnyer DM (2009) Frontal-limbic white matter pathway associations with the serotonin transporter gene promoter region (5-HTTLPR) polymorphism. J Neurosci 29:6229-6233. CrossRef Medline

Patenaude B, Smith SM, Kennedy DN, Jenkinson M (2011) A Bayesian model of shape and appearance for subcortical brain segmentation. Neuroimage 56:907-922. CrossRef Medline

Penny WD, Stephan KE, Daunizeau J, Rosa MJ, Friston KJ, Schofield TM, Leff AP (2010) Comparing families of dynamic causal models. PLoS Comput Biol 6:e1000709. CrossRef Medline

Pessoa L, McKenna M, Gutierrez E, Ungerleider LG (2002) Neural processing of emotional faces requires attention. Proc Natl Acad Sci U S A 99: 11458-11463. CrossRef Medline

Pezawas L, Meyer-Lindenberg A, Drabant EM, Verchinski BA, Munoz KE, Kolachana BS, Egan MF, Mattay VS, Hariri AR, Weinberger DR (2005) 5-HTTLPR polymorphism impacts human cingulate-amygdala interactions: a genetic susceptibility mechanism for depression. Nat Neurosci 8:828-834. CrossRef Medline

Poser BA, Versluis MJ, Hoogduin JM, Norris DG (2006) BOLD contrast sensitivity enhancement and artifact reduction with multiecho EPI: Parallel-acquired inhomogeneity-desensitized fMRI. Magnetic Resonance in Medicine 55:1227-1235. CrossRef Medline

Quirk GJ, Gehlert DR (2003) Inhibition of the amygdala: key to pathological states? Ann N Y Acad Sci 985:263-272. CrossRef Medline

Roelofs K, Minelli A, Mars RB, van Peer J, Toni I (2009) On the neural control of social emotional behavior. Social Cognitive and Affective Neuroscience 4:50-58. CrossRef Medline

Rudie JD, Shehzad Z, Hernandez LM, Colich NL, Bookheimer SY, Iacoboni M, Dapretto M (2012) Reduced functional integration and segregation of distributed neural systems underlying social and emotional information processing in autism spectrum disorders. Cereb Cortex 22:1025-1037. CrossRef Medline

Schardt DM, Erk S, Nüsser C, Nöthen MM, Cichon S, Rietschel M, Treutlein J, Goschke T, Walter H (2010) Volition diminishes genetically mediated amygdala hyperreactivity. Neuroimage 53:943-951. CrossRef Medline

Spielberger CD (1983) Manual for the State-Trait Anxiety Inventory (STAIform Y). Palo Alto: Consulting Psychologists.

Stephan KE, Kasper L, Harrison LM, Daunizeau J, den Ouden HE, Breakspear M, Friston KJ (2008) Nonlinear dynamic causal models for fMRI. Neuroimage 42:649-662. CrossRef Medline

Stephan KE, Penny WD, Moran RJ, den Ouden HE, Daunizeau J, Friston KJ (2010) Ten simple rules for dynamic causal modeling. Neuroimage 49: 3099-3109. CrossRef Medline

Strakowski SM, Adler CM, Almeida J, Altshuler LL, Blumberg HP, Chang KD, DelBello MP, Frangou S, McIntosh A, Phillips ML, Sussman JE, Townsend JD (2012) The functional neuroanatomy of bipolar disorder: a consensus model. Bipolar Disord 14:313-325. CrossRef Medline

Tzourio-Mazoyer N, Landeau B, Papathanassiou D, Crivello F, Etard O, Delcroix N, Mazoyer B, Joliot M (2002) Automated anatomical labeling of activations in SPM using a macroscopic anatomical parcellation of the MNI MRI single-subject brain. Neuroimage 15:273-289. CrossRef Medline

Volman I, Roelofs K, Koch S, Verhagen L, Toni I (2011a) Anterior prefrontal cortex inhibition impairs control over social emotional actions. Curr Biol 21:1766-1770. CrossRef Medline

Volman I, Toni I, Verhagen L, Roelofs K (2011b) Endogenous testosterone modulates prefrontal-amygdala connectivity during social emotional behavior. Cereb Cortex 21:2282-2290. CrossRef Medline 